\title{
Copious Closed Forms of Solutions for the Fractional Nonlinear Longitudinal Strain Wave Equation in Microstructured Solids
}

\author{
Haiyong Qin $\mathbb{D}^{1,2}$ Mostafa M. A. Khater $\mathbb{D}^{1,3}$ and Raghda A. M. Attia ${ }^{3,5}$ \\ ${ }^{1}$ School of Mathematics, Qilu Normal University, Jinan 250200, Shandong Province, China \\ ${ }^{2}$ School of Control Science and Engineering, Shandong University, Jinan 250061, Shandong, China \\ ${ }^{3}$ Department of Mathematics, Faculty of Science, Jiangsu University, Zhenjiang, China \\ ${ }^{4}$ Department of Mathematics, Obour Institutes, Cairo, Egypt \\ ${ }^{5}$ Department of Basic Science, Higher Technological Institute of $10^{\text {th }}$ Ramadan City, Ramadan City, Egypt \\ Correspondence should be addressed to Haiyong Qin; qhymath@hotmail.com
}

Received 13 February 2020; Accepted 20 March 2020; Published 30 April 2020

Guest Editor: Yi Qi

Copyright $\odot 2020$ Haiyong Qin et al. This is an open access article distributed under the Creative Commons Attribution License, which permits unrestricted use, distribution, and reproduction in any medium, provided the original work is properly cited.

A computational scheme is employed to investigate various types of the solution of the fractional nonlinear longitudinal strain wave equation. The novelty and advantage of the proposed method are illustrated by applying this model. A new fractional definition is used to convert the fractional formula of these equations into integer-order ordinary differential equations. Soliton, rational functions, the trigonometric function, the hyperbolic function, and many other explicit wave solutions are obtained.

\section{Introduction}

Fractional nonlinear evolution equation is one of the noticeable branches of science, particularly in recent years. Fractional calculus has a great profound physical background where it is able to formulate many various phenomena in distinct fields such as physics, mechanical engineering, economics, chemistry, signal processing, food supplement, applied mathematics, quasichaotic dynamical systems, hydrodynamics, system identification, statistics, finance, fluid mechanics, solid-state biology, dynamical systems with chaotic dynamical behavior, optical fibers, electric control theory, and economics and diffusion problems. The mathematical modeling of these phenomena will contain a fractional derivative which provides a great explanation of the nonlocal property of these models since it depends on both historical and current states of the problem in contrast with the classical calculus which depends on the current state only. Based on the importance of this kind of calculus, many definitions have been being derived such as conformable fractional derivative, fractional Riemann-Liouville derivatives, Caputo, and Caputo-Fabrizio definition [1-17]. These definitions have been being employed to convert the fractional nonlinear partial differential equations to nonlinear integer-order ordinary differential equation and then the computational and numerical schemes can be applied to get various types of solutions for these models and the examples of these schemes [18-30].

Recently, the mK method is formulated and applied to distinct physical models such as the complex Ginzburg-Landau model, the $(2+1)$-dimensional $\mathrm{KD}$ equation and $\mathrm{KdV}$ equation, fractional $(N+1)$ sinh-Gordon, biological population, equal width, modified equal width, and Duffing equations [31-40].

This method depends on a new auxiliary equation, which is equal to the Riccati equation [41]. The auxiliary equation of the $\mathrm{mK}$ method is given by

$$
\mathscr{E}^{\prime}(\varphi)=\frac{1}{\ln (\mathscr{Q})}\left[\delta \mathscr{Q}^{\mathscr{E}(\varphi)}+\varrho \mathscr{Q}^{-\mathscr{E}(\varphi)}+X\right]
$$

where $\delta, \varrho, \chi$, and $Q$ are the arbitrary constants. The Riccati equation is given by

$$
\mathscr{R}^{\prime}(\varphi)=\mathscr{A}_{0}+\mathscr{A}_{1} \mathscr{R}(\varphi)+\mathscr{A}_{2} \mathscr{R}^{2}(\varphi),
$$


where $\mathscr{A}_{0}, \mathscr{A}_{1}$, and $\mathscr{A}_{2}$ are the arbitrary constants. So, equations (1) and (2) are equal when $[\mathscr{E}(\varphi)=\mathscr{R}(\varphi)$, $\left.\chi=A_{1}, \varrho=A_{0}, \delta=A_{2}\right]$. Using this technique, it leads to the equalling of the $\mathrm{mK}$ auxiliary equation with many other analytical methods, but the $\mathrm{mK}$ method can obtain more solutions than most of them. This equivalence shows superiority, power, and productivity of the $\mathrm{mK}$ method.

In this context, the mK method is employed to construct new formulas of solutions for the fractional nonlinear longitudinal strain wave equation which is given in [42-48]:

$$
\mathscr{D}_{t t}^{29} \mathscr{M}+\mathscr{M}_{x x}-\gamma\left[\mathscr{L}_{1}\left(\mathscr{M}^{2}\right)_{x x}-\mathscr{L}_{2} \mathscr{M}_{x x x x}+\mathscr{L}_{3} \mathscr{D}_{t t}^{29}\left(\mathscr{M}_{x x}\right)\right]==0,
$$

where $\left[\gamma, \mathscr{L}_{1}, \mathscr{L}_{2}, \mathscr{L}_{3}\right]$ are the arbitrary constants.

This model is considered as one of the fundamental models in the microstructure of a material that is used to determine the elasticity, which is caused by the dissipation/ energy input in the content. This model contains nonlinear and dissipation terms which are the officials of construct the kink and shock waves. This shows the permanent form in medium points to a possible presence of dispersion or dissipation. The following definition of the $\mathscr{A} \mathscr{B} \mathscr{R}$ fractional operator [49-52] is applied to equation (3).

Definition 1. It is given in [17] that

$$
\mathscr{A} \mathscr{B} \mathscr{R} \mathscr{D}_{a+}^{\alpha} \mathscr{F}(t)=\frac{\mathscr{B}(\alpha)}{1-\alpha} \frac{\mathrm{d}}{\mathrm{d} t} \int_{a}^{t} \mathscr{F}(x) \mathscr{G}_{\alpha}\left(\frac{-\alpha(t-\alpha)^{\alpha}}{1-\alpha}\right) \mathrm{d} x,
$$

where $\mathscr{G}_{\alpha}$ is the Mittag-Leffler function defined by the following formula:

$$
\mathscr{G}_{\alpha}\left(\frac{-\alpha(t-\alpha)^{\alpha}}{1-\alpha}\right)=\sum_{n=0}^{\infty} \frac{(-\alpha / 1-\alpha)^{n}(t-x)^{\alpha n}}{\Gamma(\alpha n+1)},
$$

and $\mathscr{B}(\alpha)$ is a normalisation function. Thus,

$$
\mathscr{A} \mathscr{B} \mathscr{R} \mathscr{D}_{a+}^{\alpha} \mathscr{F}(x)=\frac{\mathscr{B}(\alpha)}{1-\alpha} \sum_{n=0}^{\infty}\left(\frac{-\alpha}{1-\alpha}\right)^{n} R L \mathscr{F}_{a}^{\alpha n} \mathscr{F}(x),
$$

leads to

$$
\begin{aligned}
\mathscr{M}(x, t) & =\mathscr{M}(\varphi), \\
\varphi & =x+\frac{c(1-\alpha) t^{-\alpha n}}{B(\alpha) \sum_{n=0}^{\infty}(-\alpha / 1-\alpha)^{n} \Gamma(1-\alpha n)},
\end{aligned}
$$

where $c$ is the arbitrary constant. This wave transformation converts equation (3) to ODE. Integrating the obtained ODEs twice with zero constant of the integration gives

$$
\mathscr{M}-\alpha \mathscr{M}^{2}+\lambda \mathscr{M}^{\prime \prime}=0,
$$

where $\left[\alpha=\gamma \mathscr{L}_{1} / c^{2}-1, \lambda=\left(\gamma\left(\mathscr{L}_{2}-c^{2} \mathscr{L}_{3}\right)\right) / c^{2}-1\right]$. Calculating the homogeneous balance value in equation (8) yields $n=2$. Thus, both equations have same general formula of solution and it is given according to the $\mathrm{mK}$ method by

$$
\begin{aligned}
\mathscr{M}(\varphi) & =\sum_{i=1}^{n} a_{i} \mathcal{Q}^{i \mathscr{E}(\varphi)}+\sum_{i=1}^{n} b_{i} \mathbb{Q}^{-i \mathscr{E}(\varphi)}+a_{0} \\
& =a_{1} \mathscr{Q}^{\mathscr{E}(\varphi)}+a_{2} \mathscr{Q}^{2 \mathscr{E}(\varphi)}+a_{0}+b_{2} \mathcal{Q}^{-2 \mathscr{E}(\varphi)}+b_{1} \mathcal{Q}^{-\mathscr{E}(\varphi)},
\end{aligned}
$$

where $a_{0}, a_{1}, a_{2}, b_{1}$, and $b_{2}$ are the arbitrary constants.

The order for the rest of this article is shown as follows: Section 2 applies the $\mathrm{mK}$ method to the nonlinear fractional strain wave equation. Section 3 discusses the obtained computational results and explains the comparison between them and that obtained in previous work. Moreover, it shows the comparison between the obtained numerical results. Section 4 gives the conclusion of the whole research.

\section{Abundant Wave Solutions of the Fractional Strain Wave Equation}

Applying the $\mathrm{mK}$ method with its auxiliary equation and the suggested general solutions for the fractional strain wave equation leads to a system of algebraic equations. Using Mathematica 11.2 to find the values of the parameters in this system leads to the following.

\subsection{Family I.}

$$
\begin{aligned}
& {\left[a_{0} \longrightarrow \frac{b_{2}\left(-\sqrt{\left(\chi^{2}-4 \delta \varrho\right)^{2}}+8 \delta \varrho+\chi^{2}\right)}{12 \varrho^{2}}, a_{1} \longrightarrow 0, a_{2} \longrightarrow 0, b_{1} \longrightarrow \frac{b_{2} \chi}{\varrho}, \alpha \longrightarrow-\frac{6 \varrho^{2}}{b_{2} \sqrt{\left(4 \delta \varrho-\chi^{2}\right)^{2}}}\right.} \\
& \left.\lambda \longrightarrow-\frac{1}{\sqrt{\left(\chi^{2}-4 \delta \varrho\right)^{2}}}, \text { where }\left(\varrho \neq 0, \chi^{2} \neq 4 \delta \varrho\right)\right]
\end{aligned}
$$


Consequently, the closed forms of solutions for the When $\left[\chi^{2}-4 \delta \varrho<0 \& \delta \neq 0\right]$, fractional strain waves model are given as follows:

$$
\begin{aligned}
\mathscr{M}_{1}(x, t)= & \frac{b_{2}}{12}\left[\frac{48 \delta^{2}}{\left(\chi-\sqrt{4 \delta \varrho-\chi^{2}} \tan \left((1 / 2) \sqrt{4 \delta \varrho-\chi^{2}}\left(x-\left((\alpha-1) c t^{-2 \alpha} / B(\alpha) \sum_{n=0}^{\infty}(-(\alpha / 1-\alpha))^{n} \Gamma(1-\alpha n)\right)\right)\right)\right)^{2}}\right. \\
& -\frac{24 \delta \chi}{\chi \varrho-\varrho \sqrt{4 \delta \varrho-\chi^{2}} \tan \left((1 / 2) \sqrt{4 \delta \varrho-\chi^{2}}\left(x-\left((\alpha-1) c t^{-2 \alpha} / B(\alpha) \sum_{n=0}^{\infty}(-(\alpha / 1-\alpha))^{n} \Gamma(1-\alpha n)\right)\right)\right)} \\
& \left.+\frac{-\sqrt{\left(\chi^{2}-4 \delta \varrho\right)^{2}}+8 \delta \varrho+\chi^{2}}{\varrho^{2}}\right], \\
\mathscr{M}_{2}(x, t)= & \frac{b_{2}}{12}\left[\frac{48 \delta^{2}}{\left(\chi-\sqrt{4 \delta \varrho-\chi^{2}} \cot \left((1 / 2) \sqrt{4 \delta \varrho-\chi^{2}}\left(x-\left((\alpha-1) c t^{-2 \alpha} / B(\alpha) \sum_{n=0}^{\infty}(-(\alpha / 1-\alpha))^{n} \Gamma(1-\alpha n)\right)\right)\right)\right)^{2}}\right. \\
& -\frac{24 \delta \chi}{\chi \varrho-\varrho \sqrt{4 \delta \varrho-\chi^{2}} \cot \left((1 / 2) \sqrt{4 \delta \varrho-\chi^{2}}\left(x-\left((\alpha-1) c t^{-2 \alpha} / B(\alpha) \sum_{n=0}^{\infty}(-(\alpha / 1-\alpha))^{n} \Gamma(1-\alpha n)\right)\right)\right)} \\
& \left.+\frac{-\sqrt{\left(\chi^{2}-4 \delta \varrho\right)^{2}}+8 \delta \varrho+\chi^{2}}{\varrho^{2}}\right] .
\end{aligned}
$$

When $\left[\chi^{2}-4 \delta \varrho>0 \& \delta \neq 0\right]$,

$$
\begin{aligned}
\mathscr{M}_{3}(x, t)= & \frac{b_{2}}{12}\left[\frac{48 \delta^{2}}{\left(\chi-\sqrt{4 \delta \varrho-\chi^{2}} \tanh \left((1 / 2) \sqrt{\chi^{2}-4 \delta \varrho}\left(x-\left((\alpha-1) c t^{-2 \alpha} / B(\alpha) \sum_{n=0}^{\infty}(-(\alpha / 1-\alpha))^{n} \Gamma(1-\alpha n)\right)\right)\right)+\chi\right)^{2}}\right. \\
& -\frac{24 \delta \chi}{\chi \varrho-\varrho \sqrt{4 \delta \varrho-\chi^{2}} \tanh \left((1 / 2) \sqrt{\chi^{2}-4 \delta \varrho}\left(x-\left((\alpha-1) c t^{-2 \alpha} / B(\alpha) \sum_{n=0}^{\infty}(-(\alpha / 1-\alpha))^{n} \Gamma(1-\alpha n)\right)\right)\right)+\chi \varrho} \\
& \left.+\frac{-\sqrt{\left(\chi^{2}-4 \delta \varrho\right)^{2}}+8 \delta \varrho+\chi^{2}}{\varrho^{2}}\right], \\
\mathscr{M}_{4}(x, t)= & \frac{b_{2}}{12}\left[\frac{48 \delta^{2}}{\left(\chi-\sqrt{4 \delta \varrho-\chi^{2}} \operatorname{coth}\left((1 / 2) \sqrt{\chi^{2}-4 \delta \varrho}\left(x-\left((\alpha-1) c t^{-2 \alpha} / B(\alpha) \sum_{n=0}^{\infty}(-(\alpha / 1-\alpha))^{n} \Gamma(1-\alpha n)\right)\right)\right)+\chi\right)^{2}}\right. \\
& \left.-\frac{24 \delta \chi}{\chi \varrho-\varrho \sqrt{4 \delta \varrho-\chi^{2}} \operatorname{coth}\left((1 / 2) \sqrt{\chi^{2}-4 \delta \varrho}\left(x-\left((\alpha-1) c t^{-2 \alpha / B}(\alpha) \sum_{n=0}^{\infty}(-(\alpha / 1-\alpha))^{n} \Gamma(1-\alpha n)\right)\right)\right)+\chi \varrho+\left(-\sqrt{\left(\chi^{2}-4 \delta \varrho\right)^{2}}+8 \delta \varrho+\chi^{2} / \varrho^{2}\right)}\right] .
\end{aligned}
$$

When $[\delta \varrho>0 \& \varrho \neq 0 \& \delta \neq 0 \& \chi=0]$,

$$
\begin{aligned}
& \mathscr{M}_{5}(x, t)=\frac{b_{2}\left(\delta \varrho\left(3 \cot ^{2}\left(\sqrt{\delta \varrho}\left(x-\left((\alpha-1) c t^{-2 \alpha} / B(\alpha) \sum_{n=0}^{\infty}(-(\alpha / 1-\alpha))^{n} \Gamma(1-\alpha n)\right)\right)\right)+2\right)-\sqrt{\delta^{2} \varrho^{2}}\right)}{3 \varrho^{2}}, \\
& \mathscr{M}_{6}(x, t)=\frac{b_{2}\left(\delta \varrho\left(3 \tan ^{2}\left(\sqrt{\delta \varrho}\left(x-\left((\alpha-1) c t^{-2 \alpha} / B(\alpha) \sum_{n=0}^{\infty}(-(\alpha / 1-\alpha))^{n} \Gamma(1-\alpha n)\right)\right)\right)+2\right)-\sqrt{\delta^{2} \varrho^{2}}\right)}{3 \varrho^{2}} .
\end{aligned}
$$


When $[\delta \varrho<0 \& \varrho \neq 0 \& \delta \neq 0 \& \chi=0]$,

$$
\begin{aligned}
& \mathscr{M}_{7}(x, t)=-\frac{b_{2}\left(3 \delta \varrho \operatorname{csch}^{2}\left(\sqrt{-\delta \varrho}\left(x-\left((\alpha-1) c t^{-2 \alpha} / B(\alpha) \sum_{n=0}^{\infty}(-(\alpha / 1-\alpha))^{n} \Gamma(1-\alpha n)\right)\right)\right)+\sqrt{\delta^{2} \varrho^{2}}+\delta \varrho\right)}{3 \varrho^{2}} \\
& \mathscr{M}_{8}(x, t)=-\frac{b_{2}\left(\delta \varrho\left(3 \tanh ^{2}\left(\sqrt{-\delta \varrho}\left(x-\left((\alpha-1) c t^{-2 \alpha} / B(\alpha) \sum_{n=0}^{\infty}(-(\alpha / 1-\alpha))^{n} \Gamma(1-\alpha n)\right)\right)\right)-2\right)+\sqrt{\delta^{2} \varrho^{2}}\right)}{3 \varrho^{2}} .
\end{aligned}
$$

When $[\chi=0 \& \varrho=-\delta]$,

$$
\begin{aligned}
\mathscr{M}_{9}(x, t)= & \frac{b_{2}}{3}\left[\frac{12}{\left(\exp \left(2 \varrho\left(x-\left((\alpha-1) c t^{-2 \alpha} / B(\alpha) \sum_{n=0}^{\infty}(-(\alpha / 1-\alpha))^{n} \Gamma(1-\alpha n)\right)\right)\right)+1\right)^{2}}\right. \\
& \left.+6 \tanh \left(\varrho\left(x-\frac{(\alpha-1) c t^{-2 \alpha}}{B(\alpha) \sum_{n=0}^{\infty}(-(\alpha / 1-\alpha))^{n} \Gamma(1-\alpha n)}\right)\right)-\frac{\sqrt{\varrho^{4}}}{\varrho^{2}}-5\right] a .
\end{aligned}
$$

When $[\chi=(\varrho / 2)=\kappa \& \delta=0]$,

$$
\begin{aligned}
\mathscr{M}_{10}(x, t)= & b_{2}\left[\frac{1}{\left(\exp \left(\kappa\left(x-\left((\alpha-1) c t^{-2 \alpha} / B(\alpha) \sum_{n=0}^{\infty}(-(\alpha / 1-\alpha))^{n} \Gamma(1-\alpha n)\right)\right)\right)-2\right)^{2}}\right. \\
& \left.+\frac{1}{2 \exp \left(\kappa\left(x-\left((\alpha-1) c t^{-2 \alpha} / B(\alpha) \sum_{n=0}^{\infty}(-(\alpha / 1-\alpha))^{n} \Gamma(1-\alpha n)\right)\right)\right)-4}-\frac{\sqrt{\kappa^{4}}}{48 \kappa^{2}}+\frac{1}{48}\right] .
\end{aligned}
$$

When $[\chi=\delta=0 \& \varrho \neq 0]$,

$$
\mathscr{M}_{11}(x, t)=\frac{b_{2}}{\varrho^{2}\left(x-\left((\alpha-1) c t^{-2 \alpha} / B(\alpha) \sum_{n=0}^{\infty}(-(\alpha / 1-\alpha))^{n} \Gamma(1-\alpha n)\right)\right)^{2}} .
$$

When $[\chi=0 \& \varrho=\delta]$,

$$
\mathscr{M}_{12}(x, t)=\frac{1}{3} b_{2}\left(3 \cot ^{2}\left(-\frac{(\alpha-1) c \varrho t^{-2 \alpha}}{B(\alpha) \sum_{n=0}^{\infty}(-(\alpha / 1-\alpha))^{n} \Gamma(1-\alpha n)}+C+x \varrho\right)-\frac{\sqrt{\varrho^{4}}}{\varrho^{2}}+2\right) .
$$

When $[\delta=0 \& \chi \neq 0 \& \varrho \neq 0]$,

$$
\begin{aligned}
\mathscr{M}_{13}(x, t)= & \frac{b_{2}}{12}\left[\frac{\chi^{2}\left(1-\left(12 \varrho / \varrho-\chi \exp \left(\chi\left(x-\left((\alpha-1) c t^{-2 \alpha} / B(\alpha) \sum_{n=0}^{\infty}(-(\alpha / 1-\alpha))^{n} \Gamma(1-\alpha n)\right)\right)\right)\right)\right)-\sqrt{\chi^{4}}}{\varrho^{2}}\right. \\
& \left.+\frac{12}{\left(\exp \left(\chi\left(x-\left((\alpha-1) c t^{-2 \alpha} / B(\alpha) \sum_{n=0}^{\infty}(-(\alpha / 1-\alpha))^{n} \Gamma(1-\alpha n)\right)\right)\right)-(\varrho / \chi)\right)^{2}}\right] .
\end{aligned}
$$

\subsection{Family II.}

$$
\begin{gathered}
{\left[a_{0} \longrightarrow \frac{\sqrt{\left(\chi^{2}-4 \delta \varrho\right)^{2}}-8 \delta \varrho-\chi^{2}}{2 \alpha \sqrt{\left(\chi^{2}-4 \delta \varrho\right)^{2}}}, a_{1} \longrightarrow-\frac{6 \delta \chi}{\alpha \sqrt{\left(\chi^{2}-4 \delta \varrho\right)^{2}}}, a_{2} \longrightarrow-\frac{6 \delta^{2}}{\alpha \sqrt{\left(4 \delta \varrho-\chi^{2}\right)^{2}}}, b_{1} \longrightarrow 0, b_{2} \longrightarrow 0\right.} \\
\left.\lambda \longrightarrow-\frac{1}{\sqrt{\left(\chi^{2}-4 \delta \varrho\right)^{2}}}, \text { where }\left(\delta \neq 0, \alpha \neq 0,4 \delta \varrho \neq \chi^{2}\right)\right]
\end{gathered}
$$


Consequently, the closed forms of solutions for the fractional strain waves model are given as follows:
When $\left[\chi^{2}-4 \delta \varrho<0 \& \delta \neq 0\right]$,

$$
\begin{aligned}
\mathscr{M}_{14}(x, t)= & {\left[3\left(\chi^{2}-4 \delta \varrho\right) \tan ^{2}\left(\frac{1}{2} \sqrt{4 \delta \varrho-\chi^{2}}\left(x-\frac{(\alpha-1) c t^{-2 \alpha}}{B(\alpha) \sum_{n=0}^{\infty}(-(\alpha / 1-\alpha))^{n} \Gamma(1-\alpha n)}\right)\right)+\sqrt{\left(\chi^{2}-4 \delta \varrho\right)^{2}}-8 \delta \varrho+2 \chi^{2}\right] } \\
& \times\left(\frac{1}{2 \alpha \sqrt{\left(\chi^{2}-4 \delta \varrho\right)^{2}}}\right), \\
\mathscr{M}_{15}(x, t)= & {\left[3\left(\chi^{2}-4 \delta \varrho\right) \cot ^{2}\left(\frac{1}{2} \sqrt{4 \delta \varrho-\chi^{2}}\left(x-\frac{(\alpha-1) c t^{-2 \alpha}}{B(\alpha) \sum_{n=0}^{\infty}(-(\alpha / 1-\alpha))^{n} \Gamma(1-\alpha n)}\right)\right)+\sqrt{\left(\chi^{2}-4 \delta \varrho\right)^{2}}-8 \delta \varrho+2 \chi^{2}\right] } \\
& \times\left(\frac{1}{2 \alpha \sqrt{\left(\chi^{2}-4 \delta \varrho\right)^{2}}}\right) .
\end{aligned}
$$

When $\left[\chi^{2}-4 \delta \varrho>0 \& \delta \neq 0\right]$,

$$
\begin{aligned}
\mathscr{M}_{16}(x, t)= & {\left[3\left(\chi^{2}-4 \delta \varrho\right) \operatorname{sech}^{2}\left(\frac{1}{2} \sqrt{\chi^{2}-4 \delta \varrho}\left(x-\frac{(\alpha-1) c t^{-2 \alpha}}{B(\alpha) \sum_{n=0}^{\infty}(-(\alpha / 1-\alpha))^{n} \Gamma(1-\alpha n)}\right)\right)+\sqrt{\left(\chi^{2}-4 \delta \varrho\right)^{2}}+4 \delta \varrho-\chi^{2}\right] } \\
& \times\left(\frac{1}{2 \alpha \sqrt{\left(\chi^{2}-4 \delta \varrho\right)^{2}}}\right), \\
\mathscr{M}_{17}(x, t)= & {\left[3\left(4 \delta \varrho-\chi^{2}\right) \operatorname{coth}^{2}\left(\frac{1}{2} \sqrt{\chi^{2}-4 \delta \varrho}\left(x-\frac{(\alpha-1) c t^{-2 \alpha}}{B(\alpha) \sum_{n=0}^{\infty}(-(\alpha / 1-\alpha))^{n} \Gamma(1-\alpha n)}\right)\right)+\sqrt{\left(\chi^{2}-4 \delta \varrho\right)^{2}}-8 \delta \varrho+2 \chi^{2}\right] } \\
& \times\left(\frac{1}{2 \alpha \sqrt{\left(\chi^{2}-4 \delta \varrho\right)^{2}}}\right) .
\end{aligned}
$$

When $\delta \varrho>0 \& \varrho \neq 0 \& \delta \neq 0 \& \chi=0$,

$$
\begin{aligned}
& \mathscr{M}_{18}(x, t)=\frac{1}{2 \alpha \sqrt{\delta^{2} \varrho^{2}}}\left[-3 \delta \varrho \sec ^{2}\left(\sqrt{\delta \varrho}\left(x-\frac{(\alpha-1) c t^{-2 \alpha}}{B(\alpha) \sum_{n=0}^{\infty}(-(\alpha / 1-\alpha))^{n} \Gamma(1-\alpha n)}\right)\right)+\sqrt{\delta^{2} \varrho^{2}}+\delta \varrho\right], \\
& \mathscr{M}_{19}(x, t)=\frac{1}{2 \alpha \sqrt{\delta^{2} \varrho^{2}}}\left[-3 \delta \varrho \csc ^{2}\left(\sqrt{\delta \varrho}\left(x-\frac{(\alpha-1) c t^{-2 \alpha}}{B(\alpha) \sum_{n=0}^{\infty}(-(\alpha / 1-\alpha))^{n} \Gamma(1-\alpha n)}\right)\right)+\sqrt{\delta^{2} \varrho^{2}}+\delta \varrho\right] .
\end{aligned}
$$


When $[\delta \varrho<0 \& \varrho \neq 0 \& \delta \neq 0 \& \chi=0]$,

$$
\begin{aligned}
& \mathscr{M}_{20}(x, t)=\frac{1}{2 \alpha}\left[\frac{\delta \varrho\left(3 \tanh ^{2}\left(\sqrt{-\delta \varrho}\left(x-\left((\alpha-1) c t^{-2 \alpha} / B(\alpha) \sum_{n=0}^{\infty}(-(\alpha / 1-\alpha))^{n} \Gamma(1-\alpha n)\right)\right)\right)-2\right)}{\sqrt{\delta^{2} \varrho^{2}}}+1\right] \\
& \mathscr{M}_{21}(x, t)=\frac{1}{2 \alpha \sqrt{\delta^{2} \varrho^{2}}}\left[3 \delta \varrho \operatorname{csch}^{2}\left(\sqrt{-\delta \varrho}\left(x-\frac{(\alpha-1) c t^{-2 \alpha}}{B(\alpha) \sum_{n=0}^{\infty}(-(\alpha / 1-\alpha))^{n} \Gamma(1-\alpha n)}\right)\right)+\sqrt{\delta^{2} \varrho^{2}}+\delta \varrho\right] .
\end{aligned}
$$

When $[\chi=0 \& \varrho=-\delta]$,

$$
\mathscr{M}_{22}(x, t)=\frac{1}{2 \alpha \sqrt{\varrho^{4}}}\left[-3 \varrho^{2} \operatorname{coth}^{2}\left(\varrho\left(x-\frac{(\alpha-1) c t^{-2 \alpha}}{B(\alpha) \sum_{n=0}^{\infty}(-(\alpha / 1-\alpha))^{n} \Gamma(1-\alpha n)}\right)\right)+\sqrt{\varrho^{4}}+2 \varrho^{2}\right] .
$$

When $[\chi=\delta=\kappa \& \varrho=0]$,

$$
\mathscr{M}_{23}(x, t)=\frac{1}{2 \alpha}\left[1-\frac{\sqrt{\kappa^{4}}\left(3 \operatorname{csch}^{2}\left((1 / 2) \kappa\left(x-\left((\alpha-1) c t^{-2 \alpha} / B(\alpha) \sum_{n=0}^{\infty}(-(\alpha / 1-\alpha))^{n} \Gamma(1-\alpha n)\right)\right)\right)+1\right)}{\kappa^{2}}\right] .
$$

When $\varrho=0 \& \chi \neq 0 \& \delta \neq 0$,

$$
\begin{aligned}
\mathscr{M}_{24}(x, t)= & \frac{-1}{2 \alpha}\left[\frac { \sqrt { \chi ^ { 4 } } } { \chi ^ { 2 } } \left(\frac{48}{\left(\delta \exp \left(\chi\left(x-\left((\alpha-1) c t^{-2 \alpha} / B(\alpha) \sum_{n=0}^{\infty}(-(\alpha / 1-\alpha))^{n} \Gamma(1-\alpha n)\right)\right)\right)-2\right)^{2}}\right.\right. \\
& \left.\left.+\frac{24}{\delta \exp \left(\chi\left(x-\left((\alpha-1) c t^{-2 \alpha} / B(\alpha) \sum_{n=0}^{\infty}(-(\alpha / 1-\alpha))^{n} \Gamma(1-\alpha n)\right)\right)\right)-2}+1\right)-1\right] .
\end{aligned}
$$

When $[\chi=0 \& \varrho=\delta]$,

$$
\mathscr{M}_{25}(x, t)=\frac{1}{2 \alpha \sqrt{\varrho^{4}}}\left[-3 \varrho^{2} \sec ^{2}\left(-\frac{(\alpha-1) c t^{-2 \alpha}}{B(\alpha) \sum_{n=0}^{\infty}(-(\alpha / 1-\alpha))^{n} \Gamma(1-\alpha n)}+C+x \varrho\right)+\sqrt{\varrho^{4}}+\varrho^{2}\right] .
$$

\section{Results and Discussion}

This section is divided into two main parts. The first part shows studying the obtained computational solutions for the fractional suggested model, while the second part presents a comparison between them and other obtained results in previous work.

(1) The solutions obtained in this paper:

(i) In this research paper, the fractional nonlinear longitudinal strain wave equation is investigated by the employment of the $\mathrm{mK}$ method and a new fractional definition $(\mathscr{A} \mathscr{B} \mathscr{R})$. Abundant explicit closed forms of solutions are obtained (twenty five solutions).

(2) The previously obtained solutions in previous work:

(i) In [53], two analytical methods were applied to three different models involving our two investigated models. However, they used two schemes but a very few special solutions were obtained.

(ii) The two analytical schemes in [53] are just a special case of the $\mathrm{mK}$ method when $\left[Q^{\mathscr{M}(\varphi)}=\right.$ $\left.\left(G^{\prime} / G\right), \varrho=-\mu, \chi=-\lambda, \delta=1\right]$.

(iii) Equation (22) is equal to equation (3.9) in [53] when $\left[e_{0}=-12 \delta(\mu+d(d-\lambda)),-3\left(\lambda^{2}-4 \mu\right)=\right.$ $\delta \lambda \varrho]$.

(iv) All other solutions obtained in this paper are considered new solutions when compared with those obtained in [53].

\section{Conclusion}

In our research paper, we solved the flaws and disadvantages of the $\left(G^{\prime} / G\right)$-expansion methods that are used in [53] by M. Ali Akbar et al., and as shown in the previous section, it is just a particular case of our applied method in this research paper. Moreover, a new definition of fractional derivative is 
used, successfully converting the fractional from our abovementioned models to integer-order ordinary differential equations. Abundance new solutions for both the models were obtained.

\section{Data Availability}

The data that support the findings of this study are available from the corresponding author upon reasonable request.

\section{Conflicts of Interest}

The authors declare that they have no conflicts of interest.

\section{Acknowledgments}

This research was supported by the National Natural Science Foundation of China, Grant nos. 61973193 and 61807010, Shandong Provincial Natural Science Foundation (Grant nos. ZR2017BA009 and ZR2016AB04), a project of Shandong Province Higher Educational Science and Technology Program (Grant no. J17KB121), and Foundation for Young Teachers of Qilu Normal University (Grant nos. 2016L0605, 2015L0603, 2017JX2311, and 2017JX2312).

\section{References}

[1] M. M. Al Qurashi, A. Yusuf, A. Yusuf, A. I. Aliyu, and M. Inc, "Optical and other solitons for the fourth-order dispersive nonlinear Schrödinger equation with dual-power law nonlinearity," Superlattices and Microstructures, vol. 105, pp. 183-197, 2017.

[2] B. Dumitru and O. P. Agrawal, "Fractional Hamilton formalism within Caputo's derivative," Czechoslovak Journal of Physics, vol. 56, no. 10-11, pp. 1087-1092, 2006.

[3] B. Dumitru and S. I. Muslih, "About Lagrangian formulation of classical fields within Riemann-Liouville fractional derivatives," in Proceedings of the ASME 2005 International Design Engineering Technical Conferences and Computers and Information in Engineering Conference, American Society of Mechanical Engineers Digital Collection, Long Beach, CA, USA, pp. 1457-1464, September 2005.

[4] A. El-Ajou, M. N. Oqielat, Z. Al-Zhour, S. Kumar, and S. Momani, "Solitary solutions for time-fractional nonlinear dispersive PDEs in the sense of conformable fractional derivative," Chaos: An Interdisciplinary Journal of Nonlinear Science, vol. 29, no. 9, Article ID 093102, 2019.

[5] E. F. D. Goufo, S. Kumar, and S. B. Mugisha, "Similarities in a fifth-order evolution equation with and with no singular kernel," Chaos, Solitons \& Fractals, vol. 130, Article ID 109467, 2020.

[6] N. Heymans and I. Podlubny, "Physical interpretation of initial conditions for fractional differential equations with Riemann-Liouville fractional derivatives," Rheologica Acta, vol. 45, no. 5, pp. 765-771, 2006.

[7] R. Hilfer, "Fractional diffusion based on riemann-liouville fractional derivatives," The Journal of Physical Chemistry B, vol. 104, no. 16, pp. 3914-3917, 2000.

[8] M. Inc, A. Yusuf, A. I. Aliyu, and D. Baleanu, "Optical soliton solutions for the higher-order dispersive cubic-quintic nonlinear Schrödinger equation," Superlattices and Microstructures, vol. 112, pp. 164-179, 2017.
[9] A. Kumar and S. Kumar, "A modified analytical approach for fractional discrete $\mathrm{KdV}$ equations arising in particle vibrations," Proceedings of the National Academy of Sciences, India Section A: Physical Sciences, vol. 88, no. 1, pp. 95-106, 2018.

[10] S. Kumar, K. Amit, S. Abbas, M. A. Qurashi, and B. Dumitru, "A modified analytical approach with existence and uniqueness for fractional Cauchy reaction-diffusion equations," Advances in Difference Equations, vol. 2020, no. 1, p. $28,2020$.

[11] S. Kumar, K. Amit, S. Momani, M. Aldhaifallah, and K. S. Nisar, "Numerical solutions of nonlinear fractional model arising in the appearance of the stripe patterns in twodimensional systems," Advances in Difference Equations, vol. 2019, no. 1, p. 413, 2019.

[12] J. Losada and J. Nieto, "Properties of a new fractional derivative without singular kernel," Progress in Fractional Differentiation and Applications, vol. 1, no. 2, pp. 87-92, 2015.

[13] Y. U. R. I. I. Luchko and R. Gorenflo, “An operational method for solving fractional differential equations with the Caputo derivatives," Acta Mathematica Vietnamica, vol. 24, no. 2, pp. 207-233, 1999.

[14] V. F. Morales-Delgado, J.-F. Gómez-Aguilar, S. Kumar, and M. A. Taneco-Hernández, "Analytical solutions of the KellerSegel chemotaxis model involving fractional operators without singular kernel," The European Physical Journal Plus, vol. 133, no. 5, p. 200, 2018.

[15] N. A. Shah and I. Khan, "Heat transfer analysis in a second grade fluid over and oscillating vertical plate using fractional caputo-fabrizio derivatives," The European Physical Journal C, vol. 76, no. 7, p. 362, 2016.

[16] F. Tchier, A. Yusuf, A. I. Aliyu, and M. Inc, "Soliton solutions and conservation laws for lossy nonlinear transmission line equation," Superlattices and Microstructures, vol. 107, pp. 320-336, 2017.

[17] A. Yusuf, M. İnç, and M. Bayram, "Soliton solutions for kudryashov-sinelshchikov equation," Sigma Journal of Engineering and Natural Sciences, vol. 37, no. 2, 2019.

[18] O. A. Arqub, A. El-Ajou, and S. Momani, "Constructing and predicting solitary pattern solutions for nonlinear timefractional dispersive partial differential equations," Journal of Computational Physics, vol. 293, pp. 385-399, 2015.

[19] V. Benci and D. F. Fortunato, "Solitary waves of the nonlinear klein-gordon equation coupled with the maxwell equations," Reviews in Mathematical Physics, vol. 14, no. 4, pp. 409-420, 2002.

[20] K. A. Gepreel and S. Omran, "Exact solutions for nonlinear partial fractional differential equations," Chinese Physics B, vol. 21, no. 11, Article ID 110204, 2012.

[21] R. Guo, "Projective synchronization of a class of chaotic systems by dynamic feedback control method," Nonlinear Dynamics, vol. 90, no. 1, pp. 53-64, 2017.

[22] J.-H. He, "Exp-function method for fractional differential equations," International Journal of Nonlinear Sciences and Numerical Simulation, vol. 14, no. 6, pp. 363-366, 2013.

[23] J.-H. He and X.-H. Wu, "Construction of solitary solution and compacton-like solution by variational iteration method," Chaos, Solitons \& Fractals, vol. 29, no. 1, pp. 108-113, 2006.

[24] M. Inc, "The approximate and exact solutions of the spaceand time-fractional Burgers equations with initial conditions by variational iteration method," Journal of Mathematical Analysis and Applications, vol. 345, no. 1, pp. 476-484, 2008.

[25] M. M. A. Khater, A. R. Seadawy, and D. Lu, "Elliptic and solitary wave solutions for Bogoyavlenskii equations system, couple Boiti-Leon-Pempinelli equations system and Time- 
fractional Cahn-Allen equation," Results in Physics, vol. 7, pp. 2325-2333, 2017.

[26] M. Kurulay and M. Bayram, "Approximate analytical solution for the fractional modified $\mathrm{KdV}$ by differential transform method," Communications in Nonlinear Science and $\mathrm{Nu}$ merical Simulation, vol. 15, no. 7, pp. 1777-1782, 2010.

[27] W. Liu and K. Chen, "The functional variable method for finding exact solutions of some nonlinear time-fractional differential equations," Pramana, vol. 81, no. 3, pp. 377-384, 2013.

[28] C. Lu, C. Fu, and H. Yang, "Time-fractional generalized Boussinesq equation for Rossby solitary waves with dissipation effect in stratified fluid and conservation laws as well as exact solutions," Applied Mathematics and Computation, vol. 327, pp. 104-116, 2018.

[29] Z. Wang and R. Guo, "Hybrid synchronization problem of a class of chaotic systems by an universal control method," Symmetry, vol. 10, no. 11, p. 552, 2018.

[30] A. Yang, L. Li, Z. Wang, and R. Guo, "Tracking control of a class of chaotic systems," Symmetry, vol. 11, no. 4, p. 568, 2019.

[31] A. A. Alderremy, R. A. M. Attia, J. F. Alzaidi, D. Lu, and M. Khater, "Analytical and semi-analytical wave solutions for longitudinal wave equation via modified auxiliary equation method and adomian decomposition method," Thermal Science, vol. 355, 2019.

[32] A. T. Ali, M. M. A. Khater, R. A. M. Attia, Abdel-Haleem Abdel-Aty, and D. Lu, "Abundant numerical and analytical solutions of the generalized formula of hirota-satsuma coupled KdV system," Chaos, Solitons \& Fractals, vol. 131, Article ID 109473, 2019.

[33] R. A. M. Attia, D. Lu, and M. M. A. Khater, "Chaos and relativistic energy-momentum of the nonlinear time fractional Duffing equation," Mathematical and Computational Applications, vol. 24, no. 1, p. 10, 2019.

[34] M. Khater, R. Attia, and D. Lu, "Modified auxiliary equation method versus three nonlinear fractional biological models in present explicit wave solutions," Mathematical and Computational Applications, vol. 24, no. 1, p. 1, 2019.

[35] M. M. A. Khater and R. A. M. Attia, D. Lu, Explicit lump solitary wave of certain interesting $(3+1)$-dimensional waves in physics via some recent traveling wave methods," Entropy, vol. 21, no. 4, p. 397, 2019.

[36] M. M. A. Khater, D. Lu, and R. A. M. Attia, "Dispersive long wave of nonlinear fractional $\mathrm{Wu}$-Zhang system via a modified auxiliary equation method," AIP Advances, vol. 9, no. 2, Article ID 025003, 2019.

[37] M. M. A. Khater, D. Lu, and R. A. M. Attia, "Lump soliton wave solutions for the $(2+1)$-dimensional Konopelchenko-Dubrovsky equation and KdV equation," Modern Physics Letters B, vol. 33, no. 18, Article ID 1950199, 2019.

[38] Li Jing, Y. Qiu, D. Lu, A. M. Raghda, and M. Khater, "Study on the solitary wave solutions of the ionic currents on microtubules equation by using the modified Khater method," Thermal Science, vol. 23, no. Suppl. 6, pp. 2053-2062, 2019.

[39] H. Qin, A. M. Raghda, M. Khater, and D. Lu, "Ample soliton waves for the crystal lattice formation of the conformable time-fractional $(N+1)$ Sinh-Gordon equation by the modified Khater method and the Painlevé property," Journal of Intelligent \& Fuzzy Systems, vol. 38, no. 3, pp. 2745-2752, 2004.

[40] H. Rezazadeh, A. Korkmaz, M. M. A. Khater, M. Eslami, D. Lu, and R. A. M. Attia, "New exact traveling wave solutions of biological population model via the extended rational sinhcosh method and the modified Khater method," Modern Physics Letters B, vol. 33, no. 28, p. 1950338, 2019.
[41] H. Rezazadeh, A. Korkmaz, M. Eslami, J. Vahidi, and R. Asghari, "Traveling wave solution of conformable fractional generalized reaction Duffing model by generalized projective Riccati equation method," Optical and Quantum Electronics, vol. 50, no. 3, p. 150, 2018.

[42] Z. Ayati, K. Hosseini, and M. Mirzazadeh, "Application of Kudryashov and functional variable methods to the strain wave equation in microstructured solids," Nonlinear Engineering, vol. 6, no. 1, pp. 25-29, 2017.

[43] A. Berezovski, J. Engelbrecht, A. Salupere, K. Tamm, T. Peets, and M. Berezovski, "Dispersive waves in microstructured solids," International Journal of Solids and Structures, vol. 50, no. 11-12, pp. 1981-1990, 2013.

[44] A. Berezovski, J. Engelbrecht, and M. Berezovski, "Waves in microstructured solids: a unified viewpoint of modeling," Acta Mechanica, vol. 220, no. 1-4, pp. 349-363, 2011.

[45] J. Engelbrecht, A. Berezovski, P. Franco, and M. Braun, "Waves in microstructured materials and dispersion," Philosophical Magazine, vol. 85, no. 33-35, pp. 4127-4141, 2005.

[46] J. . Engelbrecht and P. Franco, "Waves in microstructured solids with nonlinearities in microscale," Proceedings-Estonian Academy of Sciences Physics Mathematics, vol. 52, pp. 12-20, 2003.

[47] P. Franco, P. Cermelli, A. Porubov et al., "Nonlinear waves in 1-D solids with microstructure," Materials Chemistry and Physics, vol. 7, no. 1, pp. 9-16, 2004.

[48] A. V. Porubov, P. Franco, and G. A. Maugin, "Selection of two-dimensional nonlinear strain waves in micro-structured media," Comptes Rendus Mecanique, vol. 332, no. 7, pp. 513-518, 2004.

[49] A. Atangana and J. F. Gómez-Aguilar, "Numerical approximation of Riemann-Liouville definition of fractional derivative: from Riemann-Liouville to Atangana-Baleanu," Numerical Methods for Partial Differential Equations, vol. 34, no. 5, pp. 1502-1523, 2018.

[50] A. Atangana and I. Koca, "Chaos in a simple nonlinear system with Atangana-Baleanu derivatives with fractional order," Chaos, Solitons \& Fractals, vol. 89, pp. 447-454, 2016

[51] M. M. A. Khater and B. Dumitru, "On new analytical and semi-analytical wave solutions of the Quadratic-cubic fractional nonlinear schrödinger equation," chaos, solitons \& fractals, 2020.

[52] M. M. A. Khater and B. Dumitru, "On the new explicit computational and numerical solutions of the fractional nonlinear space-time Telegraph equation," Modern physics letter A, 2019.

[53] H. Naher and F. A. Abdullah, "New generalized and improved $\left(G^{\prime} / G\right)$-expansion method for nonlinear evolution equations in mathematical physics," Journal of the Egyptian Mathematical Society, vol. 22, no. 3, pp. 390-395, 2014. 